\title{
Document Image Binarization Using Retinex and Global Thresholding
}

\author{
Marian Wagdy ${ }^{12}$, Ibrahima Faye ${ }^{13}$ and Dayang Rohaya ${ }^{12}$ \\ Department of Computer and Information Sciences 2 \\ Department of Fundamental and Applied Sciences 3 \\ Centre of Intelligent Signal and Imaging Research 1 (CISIR) \\ Universiti Teknologi Petronas, Malaysia
}

Received 15th May 2014; revised 15th April 2015; accepted 9th Jun 2015

\begin{abstract}
Document images are usually degraded in the course of photocopying, faxing, printing, or scanning. Degradation problems seems negligible to human eyes but can be responsible for an abrupt decline in accuracy by the current generation of optical character recognition (OCR) systems. In this paper we present a binarization method based on retinex theory followed by a global threshold. The proposed method has been evaluated and compared to other existing methods. The experimental results showed high quality results in terms of visual criteria and OCR performance for the proposed method even for documents captured in an inadequate illumination condition.
\end{abstract}

Key Words:Thresholding, binarization, retinex theory,degradation, lightness, digitization, OCR systems.

\section{Introduction}

Cameras and scanners are nowadays widely used to convert grayscale or color documents into digital images. The digitization of documents makes it easier to access, preserve, share, and improve document processing times and throughput. Historical documents can be seen only from their physical location, but after digitization, they are accessible worldwide. Therefore, digitization of cultural and historical documents is an active and growing trend. The Document Digitization solution reduces the costs associated with manual document handling, storage and processing and enables better allocation of resources to more productive tasks and ultimately helps improve profit margins.

Numerous problems resulting from document digitization process cause low performance of textual information extraction techniques. For scanned documents, salt and pepper noise, touching and broken characters, and skew have long been the processing obstacles. For camera-based images, low resolution, skew, warping and border noise are the major challenges. Because of these degradation factors, researchers

Correspondence to: ibrahima_faye@petronas.com.my

Recommended for acceptance by X. Otazu

http://dx.doi.org/10.5565/rev/elcvia.648

ELCVIA ISSN: $1577-5097$

Published by Computer Vision Center / Universitat Autonoma de Barcelona, Barcelona, Spain 
develop techniques to clean-up and enhance document images to increase the recognition accuracy of document image analysis systems.

Binarization is the first step to clean up the document image by solving the degradation problems. Binarization is the process of converting a color image to a binary one by using threshold selection approaches. Each pixel of the image is classified into either white for background or black for text as shown in Fig. 1. An accurate selection of the threshold is essential to reliably separate the document image into foreground and background, especially for document images with shadows, non-uniform illumination, noise, low contrast, smear and smudge. Further processing such as character recognition and feature extraction become easier after accurate binarization. The error in the thresholding stage will propagate to all later phases.

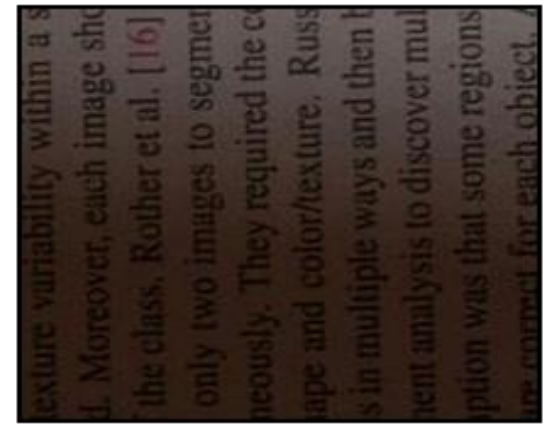

a) Original document image.

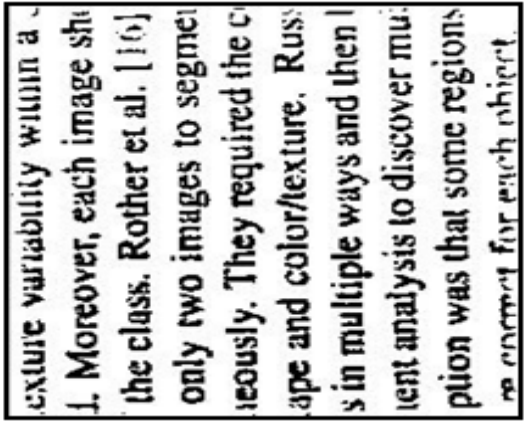

b) Binarized document image.

Figure 1: Example image for binarization process.

\section{Previous work}

Most of the binarization approaches which deal with degraded and poor quality document image can be classified into the following categories:

1. Global thresholding techniques.

2. Histogram based thresholding techniques.

3. Local thresholding techniques.

4. Clustering-based thresholding techniques.

5. Entropy-based thresholding techniques.

6. Object attribute-based methods.

7. The spatial methods.

\subsection{Global thresholding techniques}

In this category a single value threshold is selected for the whole document image. The document image is separated into foreground and background classes based on the selected threshold. Numerous global thresholding approaches have been developed in the past. In Solihin et al [1] native integral ratio (NIR) and quadratic integral ratio (QIR) are introduced. Integral ratio is a class of global thresholding method that is used to develop the two histogram-shape based methods in [1]. Gorman et al [2] proposed a global thresholding approach that is based on local features by using a connectivity-preserving measure. Gu et al [3] used morphological segmentation algorithm (differential top-hats transform) to separate the characters from background images. The drawbacks of this method include the fact that fixed parameters are used, and the method fails when the documents have variable contrast. 
Global thresholding techniques are fast and efficient to convert the grey scale document image into a binary image. However, they are unsuitable for complex and degraded documents. Moreover, global thresholding techniques produce marginal noise on the page borders when the illumination of the document is not uniform.

\subsection{Histogram-based thresholding techniques}

Histogram based techniques achieve thresholding based on the properties of the histogram shape. For example, the peaks, valleys and concavities [4] of the smoothed histogram are analyzed. Otsu's algorithm [5] is one of the most popular histogram based thresholding methods. It performs the thresholding based on a maximization criterion for the variance of the between-class pixel intensities. This method takes more time for document image binarization due to the inefficient formulation of the variance of the between-class. Moreover, the performance depends on the image types. The extension of the original method to multi-level thresholding is referred to as the Multi Otsu's method [6]. Chou et al [7] and Eikvil et al [8] developed two methods to enhance Otsu's method by dividing the documents into blocks, and then define Otsu's method for each block. Pavlidis et al [9] used grey scale pixels with significant curvature.

The common drawbacks of histogram-based techniques are their ineffectiveness for documents that do not have a bimodal histogram distribution, especially when the images contain clear handwriting on a contrasting background.

\subsection{Local thresholding techniques.}

Local based thresholding techniques evaluate different threshold for each pixel based on the grey intensities of the neighbouring pixels. The local threshold is determined according to pixel by pixel or region by region. The threshold is computed for each pixel by using some local statistics such as variance, range, or surface-fitting parameters of the neighbourhood pixels. The methods can be approached in different ways such as background subtraction [12], mean and standard derivation of pixel values [13], local mean and mean deviation [14], integral images to compute mean and variance in local windows [15]. Bernsen's [16] used the function of the lowest and highest grey values. Taxt et al [17] used Expectation-Maximization (EM). In Niblack [10], a window based local mean and standard deviation are used to calculate the threshold value. An improvement of Niblack's method is presented in Sauvola et al [11] especially when the document image is stained and badly illuminated. In this method, the threshold is calculated for each pixel instead of a window.

Some of the common disadvantages of the local based thresholding approaches include the dependence on the region size and the image characteristics, and the computational time. To combine the advantages of both global and local based thresholding techniques, hybrid approaches have been introduced as in [18].

\subsection{Clustering-based thresholding techniques}

Clustering based thresholding techniques use the grey-level samples to cluster the document image into background and foreground. The threshold is defined by using the average of the foreground and background class means. In [19], Cai et al. presented a method based on two-class Gaussian mixture models. Yanni et al [20] used another way to define the threshold by initializing the midpoint between the two assumed peaks of the histogram as $p_{-}$mid $=\left(p_{-} \max +p_{-} \min \right) / 2$, where $p_{-} \max$ is the largest nonzero gray level and $p_{-}$min is the smallest. This category fails in complex or degraded documents.

\subsection{Entropy-based thresholding techniques}

In this category, the entropies of the foreground and background regions are used as in [21]. Entropy based algorithms exploit the entropy of the distribution of the grey levels in a scene. The maximization of the entropy of the thresholded image is interpreted as indicative of maximum information transfer as in [22, 23]. Pun developed two methods [24, 25] for historical documents in which the threshold depends on the anisotropy parameter $\alpha$, which depends on the histogram asymmetry. 
The Kullback-Leibler measure is used in Li et al [26] and Tam et al [27], for selecting the threshold which minimizes the cross entropy between the thresholded image and the original image. Wong and Sahoo [28] proposed a thresholding technique based on the maximum entropy principle. Some other methods use Fuzzy entropy thresholding technique as in [29, 30]. Entropy-based thresholding techniques perform poorly in complex and degraded documents.

\subsection{Object attribute-based methods}

In this category, the threshold is computed based on some attribute quality or measuring the similarity between the binarized and the original images. These attributes can take the form of edge matching as in [31], or recurrent neural network as in [32]. Connectionist neural network and recurrent neural network are used cooperatively to determine the variables in the so-called moment-preserving equations. The designs of the networks are in such a way that the sum of square errors between the moments of the input and output images are minimized. Other techniques used fuzzy shape similarity as in [33, 34]. The index of fuzziness is used by measuring the distance between the grey-level image and its binary version. The index of fuzziness for the whole image can be obtained via the Shannon entropy measure. The optimum threshold is found by minimizing the index of fuzziness defined in terms of class (foreground, background) medians or means.

The limitations of these techniques are the dependence to image characteristics and to the region size, and the computational time.

\subsection{The spatial methods}

This class of algorithms uses higher-order probability distribution and/or correlation between pixels to define the optimal threshold. The algorithms utilize grey value distribution and also the dependency of pixels in a neighbourhood, for example, in the form of context probabilities, correlation functions, local linear dependence models of pixels, co-occurrence probabilities, 2-D entropy, etc. Rosenfeld [35] proposed the first technique exploring the spatial information. The technique considers local average of grey levels for thresholding. Others techniques have followed, using relaxation to improve on the binary map as in [36, 37]. Wesaka et al [38] made enhancement on the histogram using the Laplacian of the images. The quad tree thresholding as well as second-order statistics are used in $[39,40]$.

The common limitations in spatial-based thresholding techniques are the computational time and the inaccurate results.

\section{Proposed method}

Local thresholding methods produce high quality results but have limitation in computational time, region size dependence, and dependence on individual image characteristics. In contrast, other global thresholding methods are fast but fail when dealing with degraded documents. In the proposed method we combine the advantages of local and global thresholding techniques by using the concept of retinex theory to enhance the poor quality document images, which suffer from degradation. A global threshold technique is then used to binarized the document. The proposed method consists of two steps: degradation enhancement and binarization.

\subsection{Degradation enhancement}

For degraded and poor quality documents images, the degradation enhancement step is used to enhance the image and eliminate the noise. Retinex theory is used for this purpose.

Retinex theory is inspired by the biological phenomenon of human visual system [41]. Human visual system is able to catch the consistent colors of objects regardless of the illumination conditions. Human as example can perceives the white color of the paper under red light as a white not a red. To simulate the human visual system in the field of computer vision system, the image is decomposing into the illumination 
and the reflectance components in retinex theory [42]. Mathematically, the image in retinex theory is represented as a product of its reflectance $\mathrm{R}(\mathrm{x}, \mathrm{y})$ with its illumination $\mathrm{L}(\mathrm{x}, \mathrm{y})$, as in equation 1 :

$$
\mathrm{I}(\mathrm{x}, \mathrm{y})=\mathrm{R}(\mathrm{x}, \mathrm{y}) \cdot \mathrm{L}(\mathrm{x}, \mathrm{y})
$$

where $\mathrm{I}(\mathrm{x}, \mathrm{y})$ is the image intensity. The illumination component can be approximated by using the lowfrequency component of the measured image.

The lightness image $\mathrm{E}(\mathrm{x}, \mathrm{y})$ is the estimated reflectance of $\mathrm{R}(\mathrm{x}, \mathrm{y})$. It can be obtained by computing the ratio between the image intensity and its illumination component as in equation (2).

$$
E(x, y)=I(x, y) / L(x, y)
$$

The illumination component $\mathrm{L}(\mathrm{x}, \mathrm{y})$ is estimated by using smooth filtering with a large kernel under the assumption that the illumination component varies smoothly throughout the whole image. The proposed method uses the median filter to obtain the smooth version of the image.

Fig. 2 shows an example of a document image and its lightness version. As shown in Fig. 2.d the ratio between two adjacent points of the image and its smooth version can both detect an edge and eliminate the effect of non-uniform illumination.

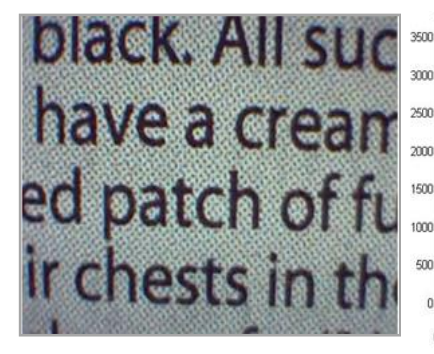

(a)

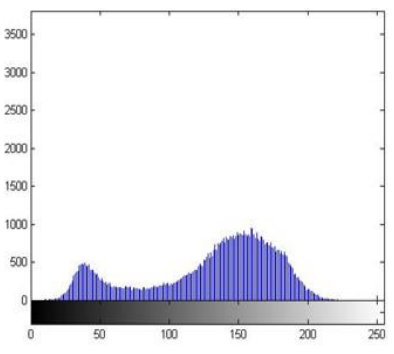

(b)

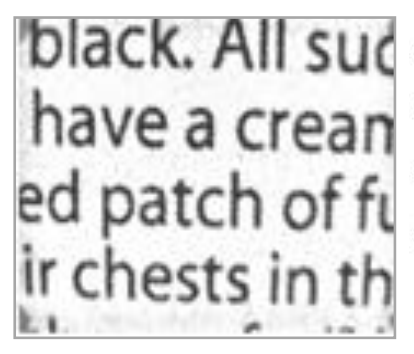

(c)

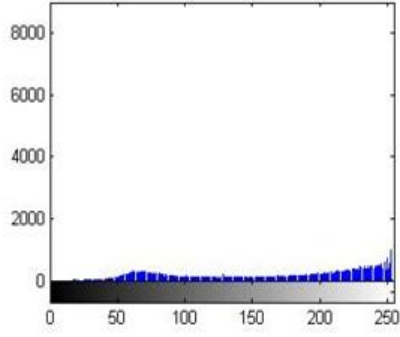

(d)

Figure 2: Example document image and its lightness version. (a) Original document image. (b) The histogram of the original document image. (c) The lightness image. (d) The histogram of the lightness image.

\subsection{Binarization}

The Retinex theory overcomes the limitation of global thresholding techniques by removing the illumination and the degradation before thresholding. The global thresholding becomes easier and more accurate after the degradation enhancement step. The most common global thresholding method in [5] is used to binarize the lightness image. Fig. 3 illustrates a comparison between the proposed method and the method in [5]. In Fig. 3.b, a large dark area appears in the results of method [5] due to the bad illumination of the original image. In Fig. 3.d, the results obtained from the proposed method are clearer and more readable for human and OCR systems.

\section{Discussion of Results}

In this section we evaluate the performance of the proposed method. The proposed method has been implemented using MATLAB R2009a and tested on a PC with Pentium Dual Core 1.8 GHz CPU.

Global threshold techniques tend to create border noise when the document image is degraded or has illumination. The proposed method overcomes this limitation by using the concept of retinex theory, which can effectively remove the illumination and the degradation before performing global thresholding. The global thresholding becomes easier and accurate after the degradation enhancement step. 


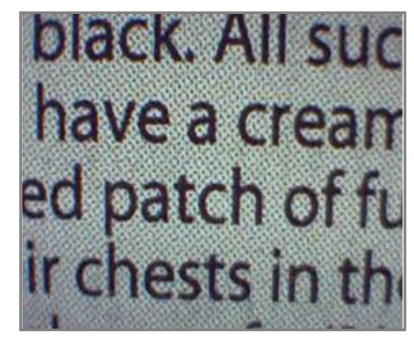

(a)

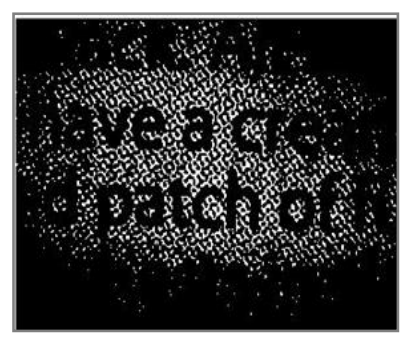

(b)

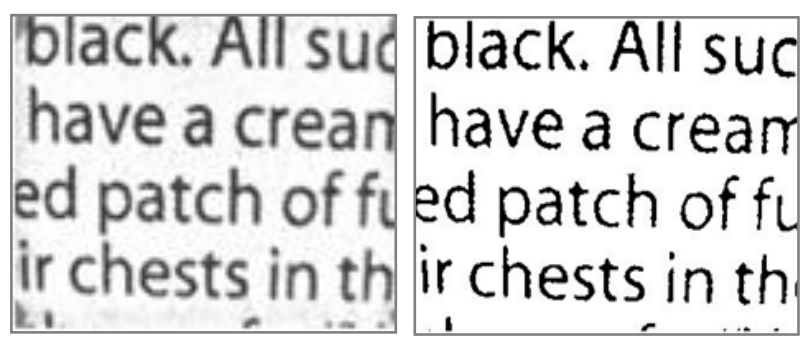

(c)

(d)

Figure 3: Binarization of degraded image. (a) Original image. (b) Binarization using Otsu method. (c) Lightness image. (d) Binarization using the proposed method.

To demonstrate the effectiveness of the proposed binarization method, it is tested on various noisy and degraded document images. Each document image illustrates the efficiency of the proposed method in addressing one of the binarization challenges such as low contrast, bad illumination, double side noise and smeared documents as shown if Fig. 4 and Fig. 5. Based on OCR performance and visual criteria, the proposed method produces high quality in all cases of degradation.
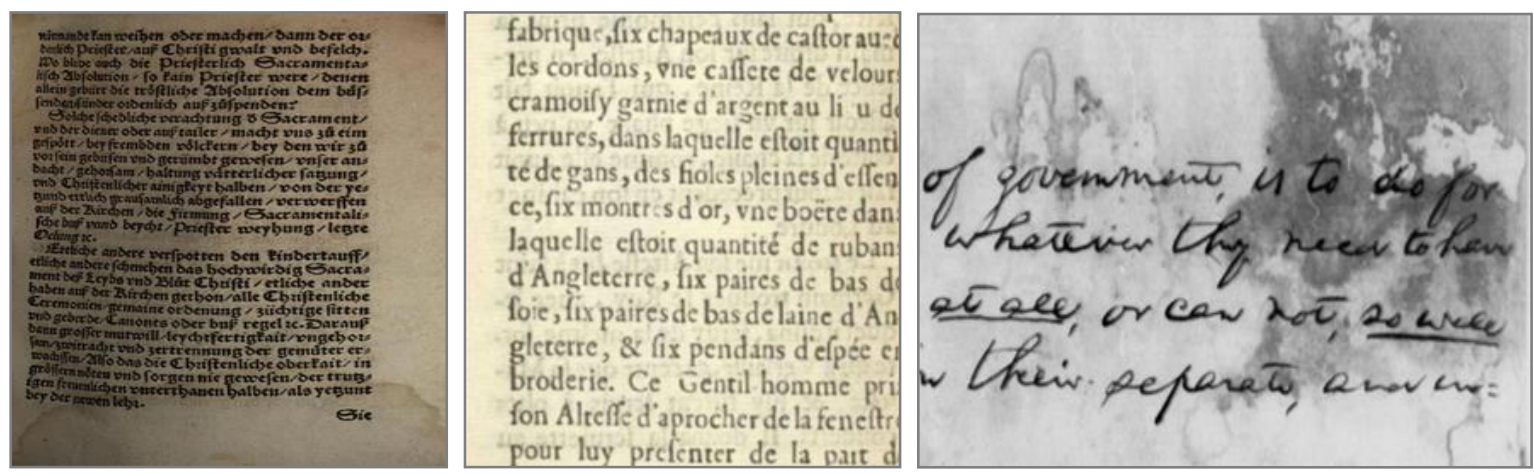

Figure 4: Example images of degradation and poor quality document images.
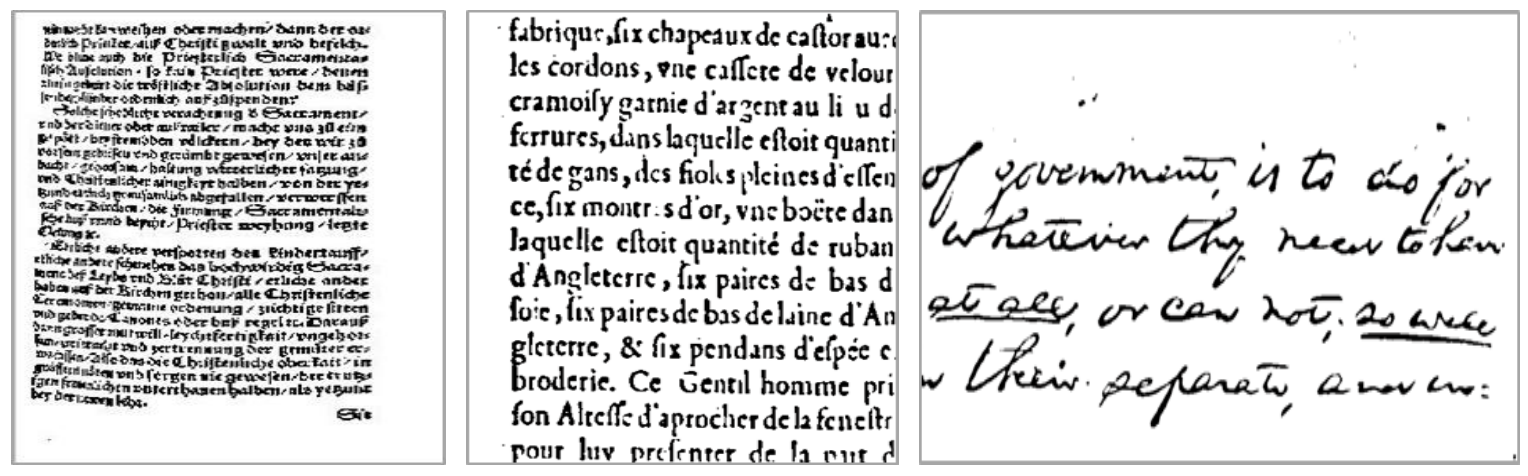

Figure 5: Example images of binarized document images.

For more experimental results the proposed method is compared with the most common eight state-of-art methods as in [7]. Three of them are global threshold methods proposed by Otsu [5], Rosenfled[4] and Pavlidis[9]; the other four are locally adaptive methods proposed by Niblack[10], Bernsen[16], Taxt[17] and Eikvil[8]; and the last was recently proposed by Chou[7]. We used the free dataset created by Chou (available in [43]). The dataset contain 122 document images photographed by an ORITE I-CAM 1300 one- 
chip color camera. The authors set the camera lens at about $6 \mathrm{~cm}$ from each document and captured a rectangular region of approximately $4.9 \times 3.4 \mathrm{~cm}$. The document images are stored as a grayscale image, consisting of $320 \times 240$ pixels. Thus, the resolution of each captured image is about 166 dots per inch.
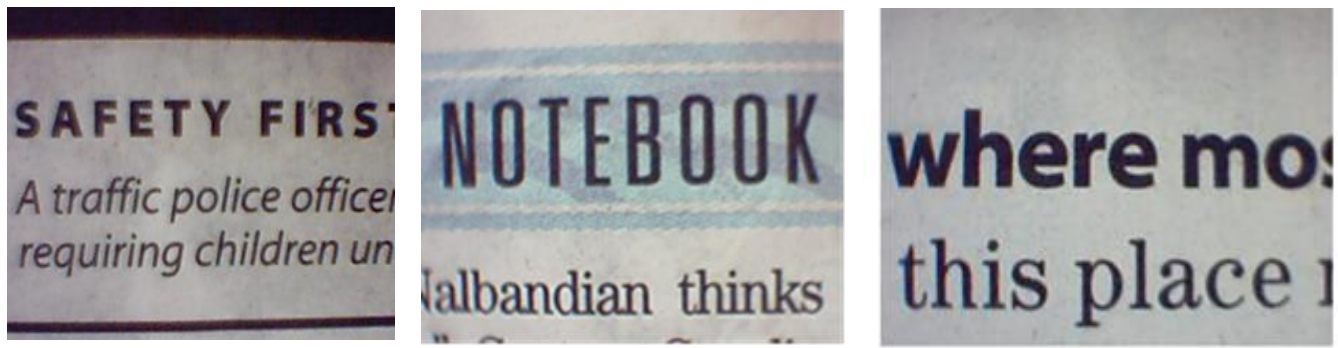

(a) Examples document images taken under the normal illumination condition.
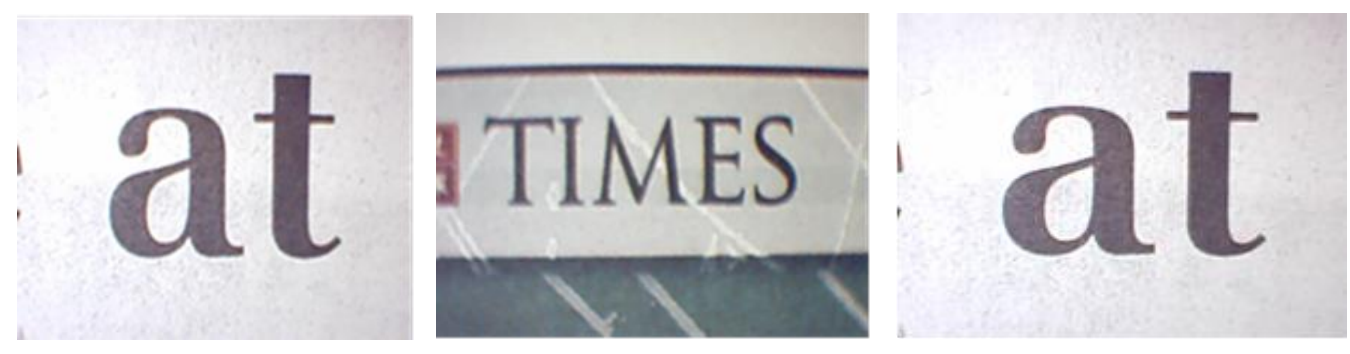

(b) Examples document images taken under the inadequate illumination condition.

Figure 6: Examples document images of Chou dataset.
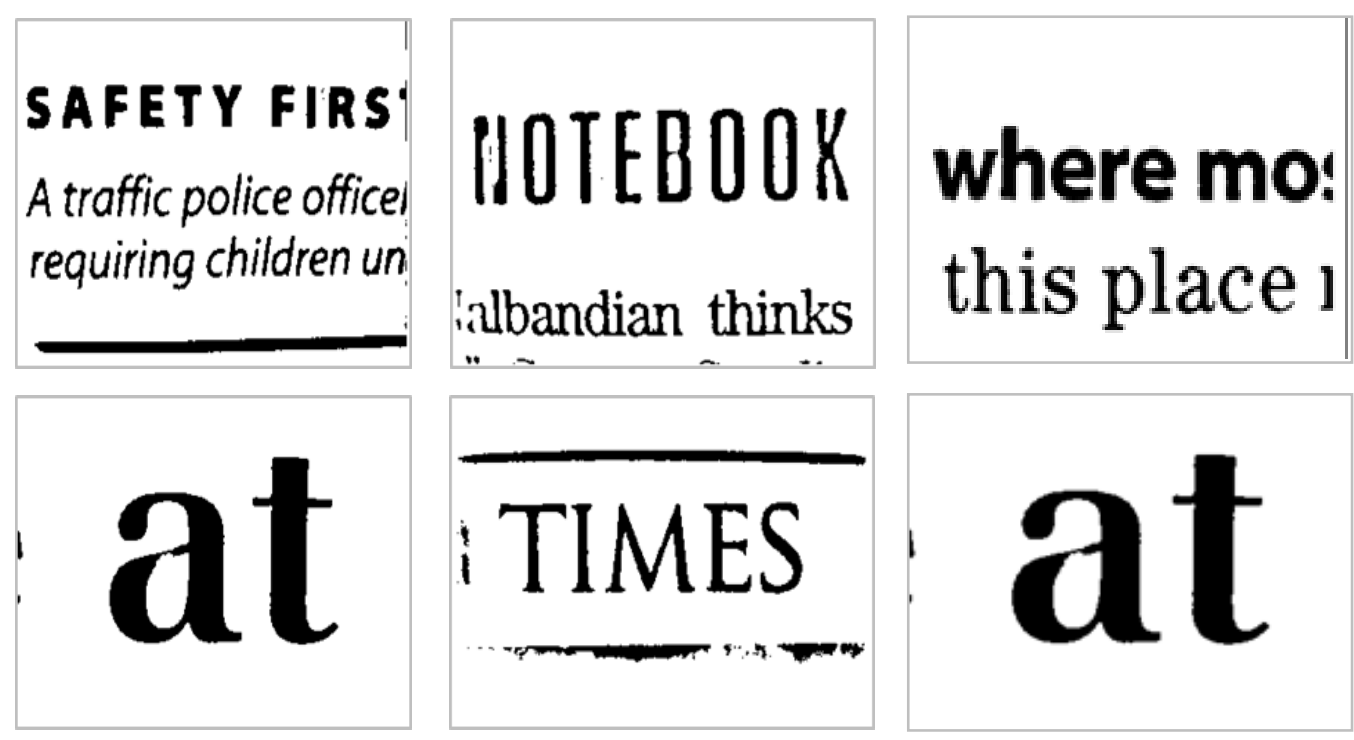

Figure 7: Binarized results of document images in figure 6.

This dataset is divided into two categories depending on the conditions:

1. The normal illumination condition.

2. The inadequate illumination condition.

The first category was photographed when the room light was on and there were no obstructions between the light and the documents, resulting in more or less uniform brightness across the images. For the second category, although the room light was on, humans or objects cast shadows over the documents, so that the shadow area appears darker than the rest of the image. In total, 60 images were produced under normal 
illumination and 62 were produced under inadequate illumination. Fig. 6 shows examples of document images of this dataset and Fig. 7 shows the binarized document images using the proposed method.

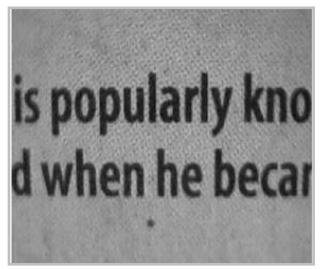

(a)

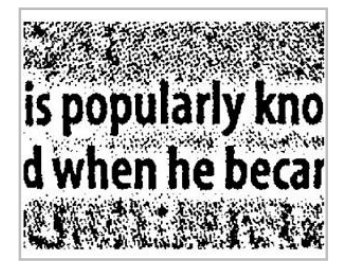

(e)

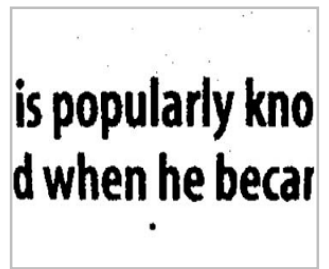

(b)

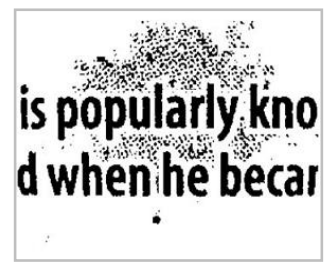

(f)

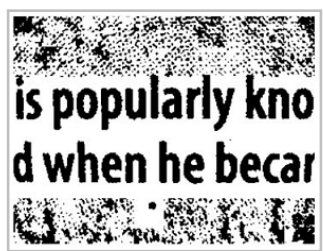

(i)

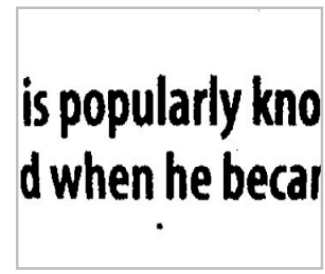

(c)

is popularly kno d when he becar

$(\mathrm{g})$

\section{is popularly kno d when he becar}

(j)

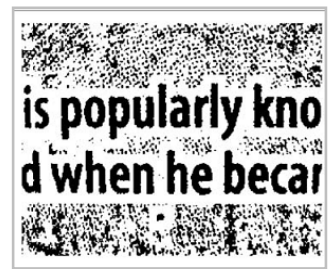

(d)

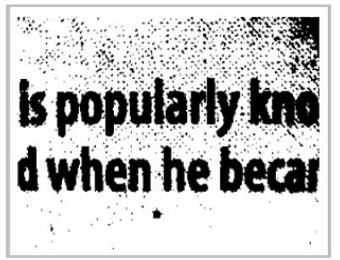

(h)

Figure 8: Binarization of poor quality document image. The image binarized by: (b) Chou, (c) Otsu, (d) Bernsen, (e) Niblack, (f) Eikvil, (g) Rosenfeld, (h) Pavlidis's, (i) Taxt and (j) our method.

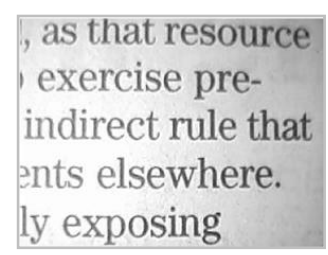

(a)

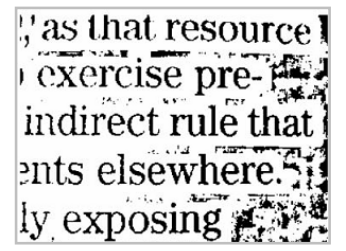

(e)

, as that resource
exercise pre-
indirect rule that
ents elsewhere.
ly exposing

(b)

, as that resource
exercise'pre-
indirect rule that
ents elsewhere.
ly exposing

(f)

, as that resource
exercise pre-
indirect rule that
ents elsewhere.
ly exposing

(i)

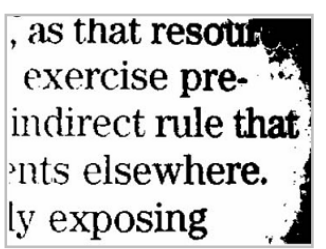

(c)

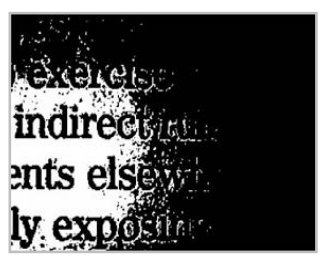

(g)

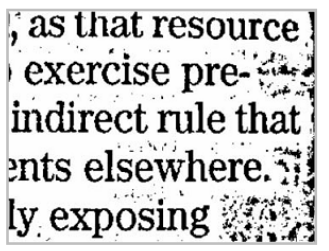

(d)

as that resource
oxercise pre-
indirect rule that
mis clsewhere.
$i$ exposing

(h)
, as that resource , exercise preindirect rule that ents elsewhere. 'v exposing

(j)

Figure 9: Binarization of poor quality document image. The image binarized by: (b) Chou, (c) Otsu, (d) Bernsen, (e) Niblack, (f) Eikvil, (g) Rosenfeld, (h) Pavlidis, (i) Taxt, and (j) our method. 
To evaluate the quality of the binarization methods, we used ABBYY Fine Reader (an OCR system). The software is used to convert scanned documents, PDF documents, and image files, including digital photos, into editable formats.

Three measures are used as in Chou[7] to evaluate the binarization method's performance on the dataset. Let $\mathrm{A}=$ the number of characters in the 122 camera taken images (there are actually 3559 characters); $\mathrm{B}=$ the number of characters detected by OCR (ABBYY) and $\mathrm{C}=$ the number of characters correctly recognized by ABBYY. The three measures, expressed in percentages, are:

1. Recall rate $=\mathrm{C} / \mathrm{A}$;

2. Precision rate $=\mathrm{C} / \mathrm{B}$;

3. $\mathrm{F}_{\beta}=\frac{\left(\beta^{2}+1\right) * \text { Recall rate } * \text { Precision rate }}{\beta^{2} *(\text { Recall rate }+ \text { Precision rate })}$

where $\beta$ is set to 1 as in [43].

Figs. 8 and 9 show examples of document images and their binarized results. The image in Figs. 8 was taken under the normal illumination condition, while the example in Fig. 9 was taken under inadequate illumination condition. To evaluate the quality of the binarization method, we used the experimental result of Chou[7]. They fed all the binarized results into the ABBYY software.

\begin{tabular}{|c|c|c|c|c|c|c|c|c|c|c|}
\hline Dataset & MES. & Chou & Otsu & Bernsen & Niblack & Eikvil & Rosenfeld & Pavlidis & Taxt & Ours \\
\hline \multirow{3}{*}{ First category } & Recall rate & 97.40 & 94.00 & 87.50 & 77.21 & 91.54 & 82.13 & 82.58 & 88.45 & 96.94 \\
\hline & Precision rate & 97.08 & 94.60 & 37.14 & 42.04 & 89.23 & 90.41 & 84.17 & 51.56 & 98.58 \\
\hline & $F_{\beta}$ & 97.24 & 94.50 & 53.12 & 54.44 & 90.37 & 86.07 & 83.37 & 65.15 & 97.76 \\
\hline \multirow{3}{*}{$\begin{array}{l}\text { Second } \\
\text { category }\end{array}$} & Recall rate & 96.84 & 84.61 & 85.31 & 85.72 & 89.06 & 57.62 & 78.03 & 89.62 & 97.88 \\
\hline & Precision rate & 96.68 & 93.10 & 44.61 & 42.53 & 78.95 & 92.15 & 79.47 & 60.67 & 100 \\
\hline & $F_{\beta}$ & 96.76 & 88.64 & 58.58 & 56.85 & 83.70 & 70.90 & 78.74 & 72.36 & 98.93 \\
\hline \multirow{3}{*}{ All dataset } & Recall rate & 97.12 & 89.31 & 86.41 & 81.47 & 90.30 & 69.88 & 80.31 & 89.04 & 97.41 \\
\hline & Precision rate & 96.88 & 93.85 & 41.38 & 42.29 & 84.09 & 91.28 & 81.82 & 56.12 & 99.29 \\
\hline & $\boldsymbol{F}_{\beta}$ & 97.00 & 91.52 & 55.96 & 55.67 & 87.04 & 79.16 & 81.06 & 68.84 & 98.34 \\
\hline
\end{tabular}

Table 1: OCR performance of the binarization methods.

Table 1 and Fig. 10 show OCR performance on the dataset for binarization methods. Based on these results, we make the following observations:

- In terms of OCR performance, our method outperforms the others methods for the whole dataset. It also produces $100 \%$ quality for OCR detection on the inadequate illumination condition.

- In the normal illumination condition, Chou's method produces a better Recall rate compared to the proposed method, but the proposed method outperforms Chou's method in remaining measures. 
- Otsu's method gives good results in the case of normal illumination condition, provided that the foreground area makes up a relatively high proportion of the image, as shown in Fig. 8.c. It fails when the images are taken under inadequate illumination conditions as in Fig. 9.c.

- Rosenfeld and Pavlidis perform well in the images that have few characters; however their performance is varying on other images.

- Although the methods of Bernsen, Taxt, and Niblack perform well in foreground areas, a pepper noise may be created in the background areas. Eikvil method performs well in background areas, but it fails if the brightness of such areas varies beyond a certain level, as shown in Fig. 8.f and Fig. 9.f.

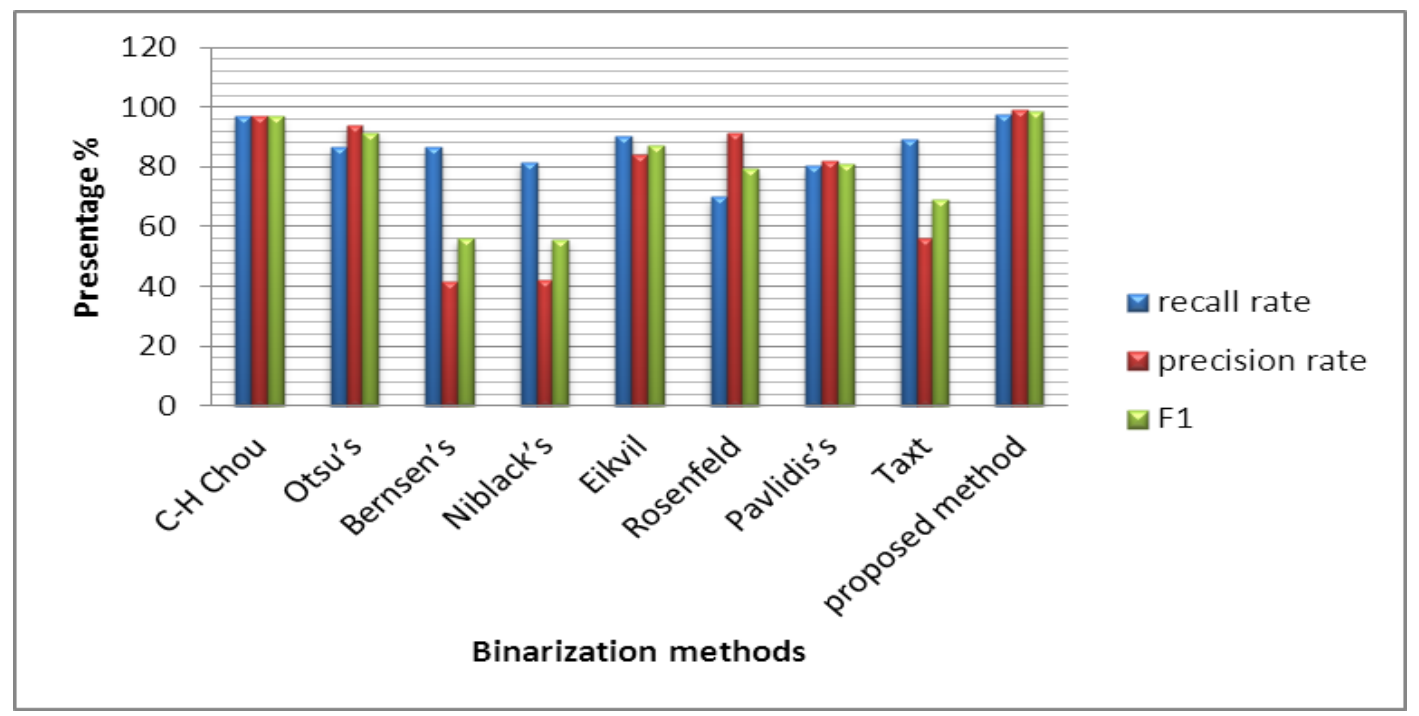

Figure 10: Comparison of binarization methods.

\section{CONCLUSION}

In this paper, we proposed a binarization technique based on the concept of retinex theory. The proposed technique combines the advantages of local and global thresholding to enhance degraded and poor quality document images. The proposed method overcomes the drawbacks of the related global threshold techniques by solving the degradation problems before binarization. From visual aspect and using OCR software, the proposed method has been evaluated and compared to other existing methods. The experimental results showed a very good performance of the proposed method even for documents captured in an inadequate illumination condition.

\section{REFERENCES}

[1] Y. Solihin and C. G. Leedham, "Integral ratio:a new class of global thresholding techniques for handwriting images"Pattern Analysis and Machine Intelligence, IEEE Transactions on, vol. 21, pp. 761-768,1999. DOI:10.1109/34.784289.

[2] L. O'Gorman, "Binarization and multithresholding of document images using connectivity" CVGIP: Graphical Models and Image Processing, vol. 56, pp. 494-506, 1994. DOI:10.1006/cgip.1994.1044.

[3] L. Gu, T. Kaneko, N. Tanaka, and R. M. Haralick, " Robust extraction of characters from color scene image using mathematical morphology" pp. 1002-1004, 1998. DOI:10.1109/ICPR.1998.711858. 
[4] A. Rosenfeld and P. De La Torre, "Histogram concavity analysis as an aid in threshold selection"Systems, Man and Cybernetics, IEEE Transactions on, pp. 231-235, 1983. DOI:10.1109/TSMC.1983.6313118.

[5] N. Otsu, "A threshold selection method from gray-level histograms"Automatica, vol. 11, pp. 23-27, 1975. DOI:10.1109/TSMC.1979.4310076.

[6] P. S. Liao, T.-S. Chen, and P.-C. Chung, "A fast algorithm for multilevel thresholding"J. Inf. Sci. Eng., vol. 17, pp. 713-727,2001. DOI:10.1.1.85.3669.

[7] C. H. Chou, W.-H. Lin, and F. Chang, "A binarization method with learning-built rules for document images produced by cameras"Pattern Recognition, vol. 43, pp. 1518-1530, 2010. DOI:10.1016/j.patcog.2009.10.016.

[8] L. Eikvil, T. Taxt, and K. Moen, "A fast adaptive method for binarization of documents images"in Proc. 1st Int. Conf. Document Analysis and Recognition, 1991. DOI:10.1016/j.patcog.2012.11.027.

[9] T. Pavlidis, "Threshold selection using second derivatives of the gray scale image"in Document Analysis and Recognition, Proceedings of the Second International Conference on, pp. 274-277, 1993. DOI:10.1109/ICDAR.1993.395733.

[10] W. Niblack, "An introduction to digital image processing "Strindberg Publishing Company, 1985. DOI:10.1002/0470035528.

[11] J. Sauvola and M. Pietikäinen, "Adaptive document image binarization"Pattern recognition, vol. 33, pp. 225-236, 2000. DOI:10.1016/S0031-3203(99)00055-2.

[12] S. Lu, B. Su, and C. L. Tan, "Document image binarization using background estimation and stroke edges"International Journal on Document Analysis and Recognition (IJDAR), vol. 13, pp. 303-314, 2010. DOI:10.1007/s10032-010-0130-8.

[13] T. Romen Singh, S. Roy, O. Imocha Singh, T. Sinam, and M. Singh, "A New Local Adaptive Thresholding Technique in Binarization" International Journal of Computer Science Issues (IJCSI), vol. 8, 2011. DOI:arXiv:1201.5227.

[14] B. Su, S. Lu, and C. L. Tan, "Binarization of historical document images using the local maximum and minimum" in Proceedings of the 9th IAPR International Workshop on Document Analysis Systems, pp. 159-166, 2010. DOI:10.1145/1815330.1815351.

[15] A. Mukherjee and S. Kanrar, "Enhancement of Image Resolution by Binarization" arXiv preprint arXiv:1111.4800, 2011. DOI:10.5120/1519-1942.

[16] J. Bernsen, "Dynamic thresholding of grey-level images"in International conference on pattern recognition, pp. 1251-1255, 1986. DOI:10.1109/ICSMC.1989.71459.

[17] T. Taxt, P. J. Flynn, and A. K. Jain, "Segmentation of document images"Pattern Analysis and Machine Intelligence, IEEE Transactions on, vol. 11, pp. 1322-1329, 1989. DOI:10.1109/ICDAR.2011.32.

[18] T. H. N. Le, T. D. Bui, and C. Y. Suen, "Ternary entropy-based binarization of degraded document images using morphological operators"in Document Analysis and Recognition (ICDAR), International Conference on, pp. 114-118, 2011. DOI:10.1109/ICPR.1998.711073.

[19] J. Cai and Z.-Q. Liu, "A new thresholding algorithm based on all-pole model"in Pattern Recognition. Proceedings. Fourteenth International Conference on, pp. 34-36.1998. DOI:10.1109/ICPR.1998.711073.

[20] M. Yanni and E. Horne, "A new approach to dynamic thresholding"in EUSIPCO'94: 9th European Conf. Sig. Process, pp. 34-44, 1994.

[21] J. Wang, E. Y. Du, C.-I. Chang, and P. D. Thouin, "Relative entropy-based methods for image thresholding" in Circuits and Systems. ISCAS. pp. II-265-II-268 vol. 2, 2002. DOI:10.1109/ISCAS.2002.1010975. 
[22] M. A. El-Sayed, "A New Algorithm Based Entropic Threshold for Edge Detection in Images" International Journal of Computer Science Issues (IJCSI), vol. 8, 2011. DOI:arXiv:1211.2500.

[23] C. Yan, N. Sang, and T. Zhang, "Local entropy-based transition region extraction and thresholding"Pattern Recognition Letters, vol. 24, pp. 2935-2941, 2003. DOI:10.1016/S01678655(03)00154-5.

[24] T. Pun, "A new method for grey-level picture thresholding using the entropy of the histogram" Signal processing, vol. 2, pp. 223-237, 1980. DOI:10.1016/0734-189X(85)90125-2.

[25] T. Pun, "Entropic thresholding, a new approach"Computer Graphics and Image Processing, vol. 16, pp. 210-239, 1981. DOI:10.1016/0146-664X(81)90038-1.

[26] C. H. Li and C. Lee, "Minimum cross entropy thresholding"Pattern Recognition, vol. 26, pp. 617-625, 1993. DOI:10.1016/0031-3203(93)90115-D.

[27] C. Li and P. K.-S. Tam, "An iterative algorithm for minimum cross entropy thresholding"Pattern Recognition Letters, vol. 19, pp. 771-776, 1998. DOI:10.1016/S0167-8655(98)00057-9.

[28] A. K. Wong and P. Sahoo, "A gray-level threshold selection method based on maximum entropy principle"Systems, Man and Cybernetics, IEEE Transactions on, vol. 19, pp. 866-871, 1989. DOI:10.1109/21.35351.

[29] A. G. Shanbhag, "Utilization of information measure as a means of image thresholding"CVGIP: Graphical Models and Image Processing, vol. 56, pp. 414-419, 1994. DOI:10.1006/cgip.1994.1037.

[30] H. Cheng, Y.-H. Chen, and Y. Sun, "A novel fuzzy entropy approach to image enhancement and thresholding"Signal Processing, vol. 75, pp. 277-301, 1999. DOI:10.1016/S0165-1684(98)00239-4.

[31] B. Gatos, I. Pratikakis, and S. J. Perantonis, "Improved document image binarization by using a combination of multiple binarization techniques and adapted edge information"in Pattern Recognition, ICPR 19th, pp. 1-4, 2008. DOI:10.1109/ICPR.2008.4761534.

[32] E. Badekas and N. Papamarkos, "Optimal combination of document binarization techniques using a self-organizing map neural network" Engineering Applications of Artificial Intelligence, vol. 20, pp. 11-24, 2007. DOI:10.1016/j.engappai.2006.04.003.

[33] C. A. Murthy and S. K. Pal, "Fuzzy thresholding: mathematical framework, bound functions and weighted moving average technique"Pattern Recognition Letters, vol. 11, pp. 197-206, 1990. DOI: 10.1016/0167-8655(90)90006-N.

[34] K. Ramar, S. Arumugam, S. Sivanandam, L. Ganesan, and D. Manimegalai, "Quantitative fuzzy measures for threshold selection"Pattern recognition letters, vol. 21, pp. 1-7, 2000. DOI:10.1016/S0167-8655(99)00120-8.

[35] R. L. Kirby and A. Rosenfeld, "A Note on the Use of (Gray Level, Local Average Gray Level) Space as an Aid in Threshold Selection" DTIC Document, 1979. DOI:10.1109/TSMC.1979.4310138.

[36] G. Fekete, J.-O. Eklundh, and A. Rosenfeld, "Relaxation: evaluation and applications" IEEE transactions on pattern analysis and machine intelligence, vol. 3, pp. 459-469, 1981. DOI:10.1109/TPAMI.1981.4767152.

[37] A. Rosenfeld and R. C. Smith, "Thresholding using relaxation: Pattern Analysis and Machine Intelligence" IEEE Transactions on, pp. 598-606, 1981. DOI: 10.1109/TSMC.1979.4310072.

[38] J. S. Weszka and A. Rosenfeld, "Histogram modification for threshold selection"vol. 9, ed: IEEE-INST ELECTRICAL ELECTRONICS ENGINEERS INC 345 E 47TH ST, NEW YORK, NY 10017-2394, pp. 38-52, 1979. DOI:10.1109/TPAMI.1982.4767203.

[39] A. Y. Wu, T.-H. Hong, and A. Rosenfeld, "Threshold selection using quadtrees" Pattern Analysis and Machine Intelligence, IEEE Transactions on, pp. 90-94, 1982. DOI:10.1109/TSMC.1978.4309892. 
[40] N. Ahuja and A. Rosenfeld, "A Note on the Use of Second-Order Gray Level Statistics for Threshold Selection" DTIC Document, 1977. DOI:10.1038/scientificamerican1277-108.

[41] E. H. Land, The retinex theory of color vision: Scientific America, 1977. DOI:10.1038/scientificamerican1277-108.

[42] Jobson, Daniel J., Z-U. Rahman, and Glenn A. Woodell. "A multiscale retinex for bridging the gap between color images and the human observation of scenes" Image Processing, IEEE Transactions on 6.7 (1997): 965-976. DOI:10.1109/83.597272.

[43] http://ocrwks11.iis.sinica.edu.tw/ dar/Download/WebPages/Binarization.htm.

[44] D. D. Lewis, "Evaluating and optimizing autonomous text classification systems" in Proceedings of the 18th annual international ACM SIGIR conference on Research and development in information retrieval, pp. 246-254, 1995. DOI:10.1145/215206.215366. 